\title{
Addressing challenges in the clinical applications associated with CRISPR/Cas9 technology and ethical questions to prevent its misuse
}

\author{
Xiang Jin Kang ${ }^{1}$, Chiong Isabella Noelle Caparas ${ }^{2}$, Boon Seng Soh ${ }^{1,2 \bowtie}$, Yong Fan ${ }^{1 \bowtie}$ \\ ${ }^{1}$ Key Laboratory for Major Obstetric Diseases of Guangdong Province, Key Laboratory of Reproduction and Genetics of \\ Guangdong Higher Education Institutes, Center for Reproductive Medicine, The Third Affiliated Hospital of Guangzhou \\ Medical University, Guangzhou 510150, China \\ 2 Disease Modeling and Therapeutics Laboratory, A*STAR Institute of Molecular and Cell Biology, 61 Biopolis Drive Proteos, \\ Singapore 138673, Singapore \\ $\bowtie$ Correspondence: bssoh@imcb.a-star.edu.sg (B. S. Soh), yongfan011@gzhmu.edu.cn (Y. Fan)
}

The recently developed RNA-guided clustered regularly interspaced short palindromic repeat (CRISPR)/CRISPR-associated 9 (Cas9) nuclease system has progressed to be an invaluable technology for genome manipulation in somatic cell types and germline model organisms. While the unprecedented advance in human embryo gene editing research has great potential in next-generation therapeutics, it raises various ethical concerns that need to be addressed before being translated for clinical use. Here, we discuss the current and potential applications of CRISPR/Cas9 technology and its limitations in clinical applications, as well as ethical and legal considerations in the treatment, disease prevention or disability in somatic cells or human embryo via gene editing.

The CRISPR/Cas9 system has been successfully utilised to introduce genetic modifications in a wide range of species, rendering it a powerful tool in genetic engineering. These applications are summarised in Table 1 below. Currently, this technology is applied in the treatment of genetic disorders in animals, but is advancing to be clinically used for the treatment of human diseases as well, specifically for those involving single gene mutations (Cox et al., 2015). Experiments to confirm that CRISPR/Cas9 technology can indeed modify pathogenic genes to treat inherited diseases have recently been carried out and reported. For example, three research groups demonstrated that the normally functioning dystrophin gene $(D m d)$ could be reintroduced in dystrophin-deficient $\mathrm{mdx}$ mice. This results in the improvement of muscle function extending from myofibers and cardiocytes, to muscle stem cells and even live animals (Barrangou and Doudna, 2016).
Additionally, several other reports have proven the in vivo application of CRISPR treatments. For example targeted genome editing via CRISPR/Cas9 enabled the expression of the wild-type Fah gene and the survival and expansion of rescued hepatocytes in adult mouse liver. Disruptions in protein convertase/subtilisin/kexin type 9 (PCSK9) also result in subsequent changes in cholesterol metabolism seen in mouse hepatocytes. Taken together, these studies demonstrated the therapeutic potential of utilising CRISPR to correct human diseases, which arise from single-gene mutations.

Another application of CRISPR/Cas9 technology for the treatment or prevention of diseases includes the modification of somatic cells. This has been demonstrated in a recentlyapproved clinical trial whereby the cells of immune system of cancer patients were genetically edited as a form of cancer therapy. At present, there are a number of human clinical trials using CRISPR against lung, prostate, and renal cell cancers. Another important current use of genome editing is in the treatment of primary HIV infection, involving the elimination of the CCR5 co-receptor via ex vivo modification. Prior to the development of the CRISPR/Cas9 system, zincfinger nuclease technology was utilised to disrupt the CCR5 co-receptor in HIV patients. This method was deemed as a promising approach for gene therapy and proceeded to evaluation for use in clinical trials (Tebas et al., 2014).

Recently, we and two other research groups have demonstrated that CRISPR/Cas9 mediated genome engineering can generate precise genetic modification or be used alongside homologous recombination to correct the 
Table 1. A summary of genetic correction using CRISPR/Cas9 technology in cell therapy, agriculture, antimicrobials, and anti-viral treatment from 2013 onwards

\begin{tabular}{|c|c|c|}
\hline Target gene & Animal model & References \\
\hline $\mathrm{RHO}$ & Rat & Bakondi et al. (2016) \\
\hline DMD & Mdx mice & Nelson et al. (2016); Long et al. (2014) \\
\hline HBB & Tripronuclear human zygotes & Liang et al. (2015) \\
\hline ASXL1 & Mouse & Valetta et al. (2015) \\
\hline PERV pol & Pig & Yang et al. (2015) \\
\hline ALB & Pig & Peng et al. (2015) \\
\hline ALS2 & Maize & Svitashev et al. (2015) \\
\hline CFTR & Human iPSCs & Firth et al. (2015) \\
\hline CCR5 & Cell lines & Li et al. (2015) \\
\hline CXCR4 & Primary human $\mathrm{CD}^{+} \mathrm{T}$ cells & Hou et al. (2015) \\
\hline FANCC & Human fibroblasts & Osborn et al. (2015) \\
\hline HBV & Mouse & Dong et al. (2015) \\
\hline $\mathrm{FAH}$ & Mouse & Yin et al. (2014) \\
\hline E6 & Human cervical cells & Yu et al. (2015) \\
\hline E7 & Human papillomavirus (HPV) positive cell lines & Hu et al. (2014) \\
\hline E. coli genome & Escherichia coli & Gomaa et al. (2014) \\
\hline APH-3 & Staphylococcus aureus & Bikard et al. (2014) \\
\hline CRYGC & Mouse & Wu et al. (2013) \\
\hline CFTR & Human intestinal stem cell organoids & Schwank et al. (2013) \\
\hline
\end{tabular}

genome of early human tripronuclear (3PN) zygotes and 2PN zygotes (Tang et al., 2017; Liang et al., 2015; Kang et al., 2016). These studies hold great promise for nextgeneration therapeutics and prospective parents who carry genetic mutations that lead to diseases, through preventing the transfer of these mutations to their offsprings.

Despite its therapeutic potential, the technical issues surrounding CRISPR technology hinder it from being clinically used. The first challenge includes off-target effects. Large genomes may consist of DNA sequences that are identical or closely resembling the target sequence, resulting in non-specific cleavage of Cas nuclease at non-target gene areas and giving rise to mutations. Depending on where the mutation is created, it may lead to cell death or transformation. Comparatively, off-target mutations are found more frequently in human cells than in mice and zebrafish (Hwang et al., 2013; Yang et al., 2013). Several papers have reported that these off-target mutations are heritable in humans as well, emphasising the need to improve CRISPR/Cas9 specificity before clinical application. As far as it is concerned, methods such as optimisation of sgRNA design, usage of paired nCas9s, paired dCas9-Fokl nucleases, and enhanced $\mathrm{Sp}$-Cas9 have been formulated to address this concern (Barrangou and Doudna, 2016; Hsu et al., 2014). Rapid advances have also been made to enhance the sensitivity of the techniques used for its evaluation throughout the whole genome. However, there remains the question of whether or not off-target effects are accountable for in a therapy that targets a single site within billions of DNA sequences, involving a large number of cells, and is designed specifically and differently among individual patients.

The second major challenge to the clinical application of gene editing is to enhance the efficiency of homology-directed recombination (HDR)-mediated precise gene modification while decreasing non-homologous end-joining (NHEJ)-induced indel production. As shown by various studies, the HDR-NHEJ ratio can be regulated by either changing the expression of machinery mediating DNA repair or optimising delivery methods and timing. A more desirable HDR-NHEJ ratio can be achieved through synchronisation of the cell cycle or utilisation of small molecules as well (Maeder and Gersbach, 2016). More recently, an endonuclease known as Cpf1 has also been shown to improve HDR. This variant not only allows the production of staggered cuts, but also needs shorter lengths of RNA (Zetsche et al., 2015).

Thirdly, there are difficulties with the delivery of Cas 9 into tissues or cells to achieve therapeutic effects. The transfection of nuclease and gRNA expression-cassette bearing plasmid DNA is acknowledged as the prominent strategy to deliver nucleases into cells. Despite being straightforward, 
this procedure is not widely used in gene and cell therapy for various reasons, including low transfection efficiency of primary cells, likelihood that plasmid fragments are randomly inserted into the gene, cytotoxicity associated with the use of DNA, and bacterial DNA sequences present in plasmid backbones (Maeder and Gersbach, 2016; Wang et al., 2016). Adeno-associated virus (AAV)-based vectors are favored for use in somatic gene therapy as they induce a mild immune response, are not pathogenic, and are capable of targeting non-dividing cells. However, these vectors are limited by their packaging limit, in which the coding sequences of Sp-Cas9, the most well-used Cas9 and its sgRNA are almost reaching (Cox et al., 2015; Maeder and Gersbach, 2016). Consequently, the development of nonDNA-dependent alternatives including pre-assembled protein-RNA complexes paved the way for novel methods for delivery in gene therapy. For example, pure nuclease proteins or Cas 9 protein-gRNA complexes are directly injected into cells. This has enabled successfully large quantities of genomic modification via electroporation, microinjections or lipid-mediated transfection. In comparison with vector-mediated nucleotide delivery methods, CRISPR-mediated genome targeting through pre-assembled protein-RNA complexes delivery methods results in improved fidelity as well as reduced cell toxicity, allowing the safety problems associated with the introduction of foreign DNA to be avoided (Maeder and Gersbach, 2016).

Finally, an issue that stems from genetic mosaicism, presumably as a result of nuclease activity succeeding the one-cell stage, still remains unresolved. Recently, Tu et al., reported that tagging Cas9 with ubiquitin-proteasomal degradation signals can facilitate the degradation of Cas9, reducing mosaic mutations and hence increases its ability to modify genomes in non-human primate embryos (Tu et al., 2017). Although problems arise from CRISPR-mediated gene editing, the technology is rapidly progressing with significant emphasis on pioneering and strategical enhancement.

With the advent of CRISPR/Cas9 technology, gene editing can be regularly and more efficiently performed in several species of organisms, ranging from insects and plants to rodents and primates. It can also be carried out in pluripotent stem cells and in human somatic cells for the purposes of basic research (Barrangou and Doudna, 2016; Baltimore et al., 2015). As CRISPR became increasingly used, research groups took genome editing a step further by inducing changes to the human germline in the hopes of correcting genetic diseases. This was first carried out in 2015 when Liang et al., demonstrated how these methods could be applied to human embryos by using CRISPR/Cas9 to cleave the endogenous beta-globing gene (HBB) off human tripronuclear zygotes, aiming to analyse the practicability and effectiveness of editing their genome to bring about therapeutic effects (Tang et al., 2017; Liang et al., 2015; Kang et al., 2016). Editing of the germline suggests that these modifications can be transmitted to successive generations, rendering it highly favorable over somatic cells if genes carrying familial disease-causing mutations could be successfully targeted. Not only will the modification of human embryo cells prevent the transmission of a known, inherited disease to the offspring from the prospective parents, it also alleviates the burden of carrying such a disease on the child (Savulescu et al., 2015).

While embryonic genomic editing has proven successful when carried out in animals, considerable technical problems must be attended to before it can be safely and predictably applied in humans as previously discussed. Referring to the study performed by Liang et al., there was a low efficiency of HDR of the endogenous $\beta$-globin gene $(H B B)$, production of mosaic embryos, and formation of offtarget mutations as shown through whole-exome sequencing and T7E1 assays (Liang et al., 2015). However, significant attention has been placed on off-target mutations especially in the context of human embryos. This is because editing of the embryo implicates that both advantageous gene corrections and detrimental effects like off-target mutations can be passed down to countless generations. As such, the use of CRISPR to genetically edit the human germline has sparked an ethical debate within the scientific community, questioning whether or not the benefits indeed outweigh the risks and raising the issue of informed consent. In this situation, informed consent of the offspring receiving the undesirable effects cannot be obtained, and there is uncertainty over who takes liability for genetic damage to be passed down several generations (Rodriguez, 2016).

Furthermore, another ethical concern arises from the risk of using CRISPR/Cas9 for non-therapeutic purposes such as enhancement. Preferred phenotypic characteristics could be achieved through genome editing of somatic or germ cells, going one step closer towards "designer babies". For example, athletic performance or intellectual abilities may be improved, posing social problems if only specific individuals are able to receive this enhancement. Once again it also raises the issue of informed consent (Rodriguez, 2016). To prevent the abuse of CRISPR/Cas9 technology, clear regulations must be placed as guidance to draw the line between the ethical and socially acceptable use of gene editing and its misuse.

In light of addressing these issues, the International Summit on Human Gene Editing in Washington DC has been held to evaluate the technical concerns, and impacts of genome editing on the society and scientific research alike. Taking into account both the advantages and downsides of embryo gene editing research, the committee has concluded that such research will not be prohibited, although extreme caution must be exercised when utilising it. The use of germline editing in research trials can potentially be conducted, however prior to approval for clinical trials, further evaluation and progress must be made such that the benefits greatly outweigh the risks. Nonetheless, embryo gene modification must only be carried out under strict regulations (http://nationalacademies.org/gene-editing/consensus- 
study/index.htm). In this regard, we advocate for the application of both international guidelines and national policies to supervise and monitor research on human embryo gene editing. While common scientific and technical difficulties are encountered by the healthcare industry and academia, the differing cultural and economical backgrounds of each country subjects it to varying ethical issues. Therefore international guidelines are placed merely to answer consensus questions and as a basis so that individual countries legislate the appropriate, and specific respective oversight policies (Bosley et al., 2015).

Being relatively new, CRISPR/Cas9 technology has not yet been exploited to its maximum capabilities. However, it has already revolutionised genomic research, significantly affecting biomedical research and delivering the clinical advantages that previous treatments could not offer. In the future, use of CRISPR for genome editing could reduce the global burden of incurable diseases such as genetic disorders, cancer or HIVIAIDS, and potentially benefiting millions of people worldwide. Although the technology is predicted to advance further, various amendments must be made to make it more specific, safe, and efficient. There also remains the risk of improper use, raising ethical concerns that stir up doubt within the society. Aside from the regulation of use, other societal concerns include ensuring that future benefits outweigh the risks, policy decisions consider the values of the society and that the diverse perspective of individuals, countries, and cultures are respected. These factors affect the conditions and circumstances in which CRISPR/Cas9 will be used.

Prior to the publication of the above-mentioned papers on human embryonic genomic editing, commentaries have also been published by prestigious journals including Nature and Science, greatly discouraging this type of research and moratorium for ethical and legal reasons (Baltimore et al., 2015; Lanphier et al., 2015). While opinions within the scientific community are divided with some supporting, and others encouraging the prohibition of research on genome modification without providing convincing reasons, we strongly felt that a complete ban will not only hinder the development of future treatments, it is unfeasible as well, especially considering the high accessibility of CRISPR/ Cas9 technology. The consequences of imposing such a ban remain questionable especially the impact on disabled people both in the present and future (Lanphier et al., 2015). Disabled people will not be protected by laws that differentiate on the grounds of disease labels. However, the views of disability groups and others that strive to protect the rights of these people and improve their social situation are often overlooked. Their opinions on the use of genetic approaches that intervene somatic and germ cells are neglected as well.

In summary, while genome editing using CRISPR-Cas9 allows for personalised medicine as well as the correction of human genetic diseases such as cystic fibrosis, several ethical considerations would need to be resolved prior to its clinical applications. The side effects of germline editing have often been cited as a justification for the cessation of its use on human germ cells. Arguments for embryonic gene modification supports its clinical use despite the suggested side effects due to the overall benefit that preventing the transmission of heritable genetic diseases brings. In addition, research on embryonic gene editing could also be expanded for non-therapeutic uses, provided that it is solely for research purposes. This is especially applicable for CRISPR-Cas9 and other germline editing technologies that may be used to resolve non-clinical scientific problems. In this regard, the US National Academies of Sciences, Engineering, and Medicine has recently made recommendations to maximize the benefits to human health of any applications of genome editing (http://nationalacademies. org/gene-editing/consensus-study/index.htm). While heritable germline editing is still underway, such clinical application is plausible in the foreseeable future. In fact, a recent paper by Hong et al. in 2017 proves that much improvement has already been made in the technology within the past 4 years. The paper reports the effective, specific, high HDR CRISPR/ Cas9-mediated correction of the heterozygous Myosinbinding protein $\mathrm{C}$ (MYBPC3) pathogenic mutation involved in the pathogenesis of hypertrophic cardiomyopathy. Genome editing was carried out in human pre-implantation embryos, resulting in embryos without mosaicism and off-target mutations (Ma et al., 2017). More researches must be carried out to evaluate the reproducibility of the technique and before proceeding with its clinical application. Nonetheless, this proves that CRISPR/Cas9 has the ability to effectively and safely target heritable germline mutations to correct genetic diseases.

At the moment, it is certain that the CRISPR/Cas9 technology itself does not pose a threat, and the breakthroughs this technology can bring in therapeutics provides further support for its use in genetic editing of somatic cells. Moving forward, due to the rapid improvement of CRISPR/Cas9 technology, continual assessment and discussions will be necessary from time to time to ensure that this technology is utilised safely and responsibly.

\section{ACKNOWLEDGEMENTS}

This work was supported by the National Natural Science Foundation of China (Grant Nos. 81370766, 81570101, and 81728002), Guangdong Province Science and Technology Project (2014TQ01R 683,2014 A02011029, 2017A020214005). The authors apologize for the omission of additional applications of CRISPR/Cas9 or citations due to the reference limitations.

\section{OPEN ACCESS}

This article is distributed under the terms of the Creative Commons Attribution 4.0 International License (http://creativecommons.org/ licenses/by/4.0/), which permits unrestricted use, distribution, and reproduction in any medium, provided you give appropriate credit to the original author(s) and the source, provide a link to the Creative Commons license, and indicate if changes were made. 


\section{REFERENCES}

Bakondi B, Lv W et al (2016) In vivo CRISPR/Cas9 gene editing corrects retinal dystrophy in the $\$ 334$ ter-3 rat model of autosomal dominant retinitis pigmentosa. Mol Ther 24(3):556-563

Baltimore D, Berg P et al (2015) Biotechnology. A prudent path forward for genomic engineering and germline gene modification. Science 348(6230):36-38

Barrangou R, Doudna JA (2016) Applications of CRISPR technologies in research and beyond. Nat Biotechnol 34(9):933-941

Bikard D, Euler CW et al (2014) Exploiting CRISPR-Cas nucleases to produce sequence-specific antimicrobials. Nat Biotechnol 32 (11):1146-1150

Bosley KS, Botchan M et al (2015) CRISPR germline engineeringthe community speaks. Nat Biotechnol 33(5):478-486

Cox DB, Platt RJ et al (2015) Therapeutic genome editing: prospects and challenges. Nat Med 21(2):121-131

Dong C, Qu L et al (2015) Targeting hepatitis B virus cccDNA by CRISPR/Cas9 nuclease efficiently inhibits viral replication. Antivir Res 118:110-117

Firth AL, Menon T et al (2015) Functional gene correction for cystic fibrosis in lung epithelial cells generated from patient iPSCs. Cell Rep 12(9):1385-1390

Gomaa AA, Klumpe HE et al (2014) Programmable removal of bacterial strains by use of genome-targeting CRISPR-Cas systems. mBio 5(1):e00913-e00928

Hou P, Chen S et al (2015) Genome editing of CXCR4 by CRISPR/cas9 confers cells resistant to HIV-1 infection. Sci Rep. 5:15577

Hsu PD, Lander ES et al (2014) Development and applications of CRISPR-Cas9 for genome engineering. Cell 157(6):1262-1278

$\mathrm{Hu}$ Z, Yu L et al (2014) Disruption of HPV16-E7 by CRISPR/Cas system induces apoptosis and growth inhibition in HPV16 positive human cervical cancer cells. Biomed Res Int 2014:612823

Hwang WY, Fu Y et al (2013) Efficient genome editing in zebrafish using a CRISPR-Cas system. Nat Biotechnol 31(3):227-229

Kang X, He W et al (2016) Introducing precise genetic modifications into human 3PN embryos by CRISPR/Cas-mediated genome editing. J Assist Reprod Genet 33(5):581-588

Lanphier E, Urnov $F$ et al (2015) Don't edit the human germ line. Nature 519(7544):410-411

Li C, Guan X et al (2015) Inhibition of HIV-1 infection of primary $\mathrm{CD}^{+}$T-cells by gene editing of CCR5 using adenovirusdelivered CRISPR/Cas9. J Gen Virol 96(8):2381-2393

Liang P, Xu Y et al (2015) CRISPR/Cas9-mediated gene editing in human tripronuclear zygotes. Protein Cell 6(5):363-372

Long C, McAnally JR et al (2014) Prevention of muscular dystrophy in mice by CRISPR/Cas9-mediated editing of germline DNA. Science 345(6201):1184-1188

$\mathrm{Ma} \mathrm{H}$, Marti-Gutierrez N et al (2017) Correction of a pathogenic gene mutation in human embryos. Nature 548(7668):413-419

Maeder ML, Gersbach CA (2016) Genome-editing Technologies for Gene and Cell Therapy. Mol Ther 24(3):430-446
Nelson CE, Hakim $\mathrm{CH}$ et al (2016) In vivo genome editing improves muscle function in a mouse model of Duchenne muscular dystrophy. Science 351(6271):403-407

Osborn MJ, Gabriel R et al (2015) Fanconi anemia gene editing by the CRISPR/Cas9 system. Hum Gene Ther 26(2):114-126

Peng J, Wang $Y$ et al (2015) Production of human albumin in pigs through CRISPR/Cas9-mediated knockin of human CDNA into swine albumin locus in the zygotes. Sci Rep 5:16705

Rodriguez E (2016) Ethical issues in genome editing using Crispr/ Cas9 system. J Clin Res Bioeth 7(2):266

Savulescu J, Pugh J et al (2015) The moral imperative to continue gene editing research on human embryos. Protein Cell 6(7):476479

Schwank G, Koo BK et al (2013) Functional repair of CFTR by CRISPR/Cas9 in intestinal stem cell organoids of cystic fibrosis patients. Cell Stem Cell 13(6):653-658

Svitashev S, Young JK et al (2015) Targeted mutagenesis, precise gene editing, and site-specific gene insertion in maize using Cas9 and guide RNA. Plant Physiol 169(2):931-945

Tang L, Zeng Y et al (2017) CRISPR/Cas9-mediated gene editing in human zygotes using Cas9 protein. Mol Genet Genomics 292 (3):525-533

Tebas P, Stein D et al (2014) Gene editing of CCR5 in autologous CD4 T cells of persons infected with HIV. N Engl J Med 370 (10):901-910

Tu Z, Yang W et al (2017) Promoting Cas9 degradation reduces mosaic mutations in non-human primate embryos. Sci Rep 7:42081

Valletta S, Dolatshad H et al (2015) ASXL1 mutation correction by CRISPR/Cas9 restores gene function in leukemia cells and increases survival in mouse xenografts. Oncotarget 6 (42):44061-44071

Wang $\mathrm{H}$, La Russa M et al (2016) CRISPR/Cas9 in genome editing and beyond. Annu Rev Biochem 85:227-264

Wu YLD, Wang Y et al (2013) Correction of a genetic disease in mouse via use of CRISPR-Cas9. Cell Stem Cell 13(6):659-662

Yang $\mathrm{H}$, Wang $\mathrm{H}$ et al (2013) One-step generation of mice carrying reporter and conditional alleles by CRISPR/Cas-mediated genome engineering. Cell 154(6):1370-1379

Yang L, Guell M et al (2015) Genome-wide inactivation of porcine endogenous retroviruses (PERVs). Science 350(6264):11011104

Yin H, Xue W et al (2014) Genome editing with Cas9 in adult mice corrects a disease mutation and phenotype. Nat Biotechnol 32 (6):551-553

Yu L, Wang X et al (2015) Disruption of human papillomavirus 16 E6 gene by clustered regularly interspaced short palindromic repeat/ Cas system in human cervical cancer cells. OncoTargets Ther 8:37-44

Zetsche B, Gootenberg JS et al (2015) Cpf1 is a single RNA-guided endonuclease of a class 2 CRISPR-Cas system. Cell 163 (3):759-771 